\title{
Complete genome sequence of Cellulophaga algicola type strain (IC166 ${ }^{\mathrm{T}}$ )
}

\author{
Birte Abt ${ }^{1}$, Megan Lu' ${ }^{2,3}$, Monica Misra ${ }^{2,3}$, Cliff Han $^{2,3}$, Matt Nolan ${ }^{2}$, Susan Lucas ${ }^{2}$, Nancy \\ Hammon $^{2}$, Shweta Deshpande ${ }^{2}$, Jan-Fang Cheng ${ }^{2}$, Roxane Tapia ${ }^{2,3}$, Lynne Goodwin ${ }^{2,3}$, Sam \\ Pitluck $^{2}$, Konstantinos Liolios², Ioanna Pagani ${ }^{2}$, Natalia Ivanova ${ }^{2}$, Konstantinos Mavromatis ${ }^{2}$, \\ Galina Ovchinikova ${ }^{2}$, Amrita Pati ${ }^{2}$, Amy Chen ${ }^{4}$, Krishna Palaniappan ${ }^{4}$, Miriam Land ${ }^{2,5}$, Loren \\ Hauser $^{2,5}$, Yun-Juan Chang ${ }^{2,5}$, Cynthia D. Jeffries ${ }^{2,5}$, John C. Detter ${ }^{2,3}$, Evelyne Brambilla ${ }^{1}$, \\ Manfred Rohde ${ }^{6}$, Brian J. Tindall ${ }^{1}$, Markus Göker ${ }^{1}$, Tanja Woyke ${ }^{2}$, James Bristow ${ }^{2}$, Jonathan \\ A. Eisen ${ }^{2,7}$, Victor Markowitz ${ }^{4}$, Philip Hugenholtz ${ }^{2,8}$, Nikos C. Kyrpides ${ }^{2}$, Hans-Peter Klenk', \\ and Alla Lapidus ${ }^{2 *}$ \\ ${ }^{1}$ DSMZ - German Collection of Microorganisms and Cell Cultures GmbH, Braunschweig, \\ Germany \\ ${ }^{2}$ DOE Joint Genome Institute, Walnut Creek, California, USA \\ ${ }^{3}$ Los Alamos National Laboratory, Bioscience Division, Los Alamos, New Mexico, USA \\ ${ }^{4}$ Biological Data Management and Technology Center, Lawrence Berkeley National \\ Laboratory, Berkeley, California, USA \\ ${ }^{5}$ Oak Ridge National Laboratory, Oak Ridge, Tennessee, USA \\ ${ }^{6} \mathrm{HZI}$ - Helmholtz Centre for Infection Research, Braunschweig, Germany \\ ${ }^{7}$ University of California Davis Genome Center, Davis, California, USA \\ ${ }^{8}$ Australian Centre for Ecogenomics, School of Chemistry and Molecular Biosciences, The \\ University of Queensland, Brisbane, Australia \\ *Corresponding author: Alla Lapidus
}

Keywords: aerobic, motile by gliding, Gram-negative, agarolytic, chemoorganotrophic, cold adapted enzymes, Flavobacteriaceae, GEBA

Cellulophaga algicola Bowman 2000 belongs to the family Flavobacteriaceae within the phylum 'Bacteroidetes' and was isolated from Melosira collected from the Eastern Antarctic coastal zone. The species is of interest because its members produce a wide range of extracellular enzymes capable of degrading proteins and polysaccharides with temperature optima of $20-30^{\circ} \mathrm{C}$. This is the first completed genome sequence of a member of the genus Cellulophaga. The 4,888,353 bp long genome with its 4,285 protein-coding and 62 RNA genes consists of one circular chromosome and is a part of the Genomic Encyclopedia of Bacteria and Archaea project.

\section{Introduction}

Strain IC166 ${ }^{\mathrm{T}}$ (= DSM $14237=$ CIP $107446=$ LMG 21425 ) is the type strain of Cellulophaga algicola, which belongs to the family Flavobacteriaceae within the phylum 'Bacteroidetes'. The strain was isolated from the surface of the chain-forming seaice diatom Melosira collected from the Eastern Antarctic coastal zone, and was described by Bowman in 2000 [1]. Currently, there are six species placed in the genus Cellulophaga, namely C. algicola [1], C. baltica, C. fucicola, C. lytica [2], C. pacifica [3] and C. tyrosinoxydans [4]. C. lytica is the type species of the genus Cellulophaga [2]. The generic name of the genus derives from the Neo-Latin word 'cellulosum' meaning 'cellulose' and the Greek word 'phagein' meaning 'to eat', referring to an eater of cellulose. Here we present a summary classification and a set of features for C. algicola IC166 ${ }^{\mathrm{T}}$, together with the description of the complete genomic sequencing and annotation.

\section{Classification and features}

A representative genomic $16 \mathrm{~S}$ rRNA sequence of $C$. algicola was compared using NCBI BLAST under default settings (e.g., considering only the highscoring segment pairs (HSPs) from the best 250 
hits) with the most recent release of the Greengenes database [5] and the relative frequencies, weighted by BLAST scores, of taxa and keywords (reduced to their stem [6]) were determined. The five most frequent genera were Cellulophaga (39.5\%), Maribacter (7.8\%), Flavobacterium (5.6\%), Cytophaga (5.4\%) and Formosa (4.7\%) (135 hits in total). Regarding the 21 hits to sequences from members of the species, the average identity within HSPs was $95.8 \%$, whereas the average coverage by HSPs was $94.9 \%$. Regarding the 16 hits to sequences from other members of the genus, the average identity within HSPs was 94.7\%, whereas the average coverage by HSPs was $94.7 \%$. Among all other species, the one yielding the highest score was $C$. baltica, which corresponded to an identity of $98.1 \%$ and a HSP coverage of $97.8 \%$. The highest-scoring environmental sequence was GU452686 ('sediments coast oil polluted Black Sea coastal sediment clone 70SZ2'), which showed an identity of $96.5 \%$ and a HSP coverage of $98.1 \%$. The five most frequent keywords within the labels of environmental samples which yielded hits were 'marin' (4.7\%), 'water' (4.3\%), 'sediment' (4.3\%), 'sea' (3.5\%) and 'coastal' (2.6\%) (115 hits in total). Environmental samples which yielded hits of a higher score than the highest scoring species were not found.

The environmental samples database (env_nt) contains the marine metagenome clone ctg_1101667042524 (AACY022635173) isolated from Sargasso Sea near Bermuda, sharing 92\% identity with IC166 ${ }^{\mathrm{T}}$ [7] (as of January 2011).

Figure 1 shows the phylogenetic neighborhood of C. algicola $\mathrm{IC} 166^{\mathrm{T}}$ in a $16 \mathrm{~S}$ rRNA based tree. The sequences of the five 16S rRNA gene copies in the genome differ from each other by up to two nucleotides, and differ by up to 14 nucleotides from the previously published 16S rRNA sequence (AF001366), which contains nine ambiguous base calls.

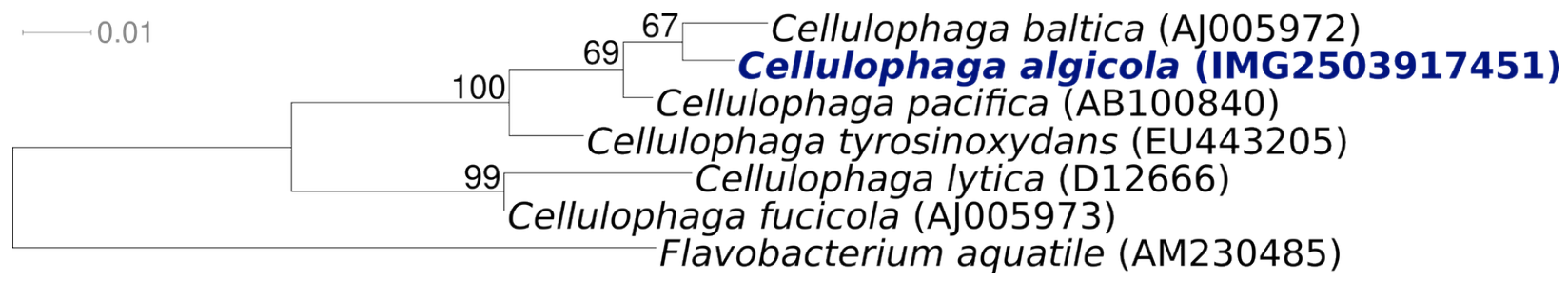

Figure 1. Phylogenetic tree highlighting the position of $C$. algicola IC $166^{\top}$ relative to the other type strains within the family Flavobacteriaceae. The tree was inferred from 1,458 aligned characters [8,9] of the 16S rRNA gene sequence under the maximum likelihood criterion [10] and rooted in accordance with the current taxonomy. The branches are scaled in terms of the expected number of substitutions per site. Numbers above branches are support values from 350 bootstrap replicates [11] if larger than 60\%. Lineages with type strain genome sequencing projects registered in GOLD [12] are shown in blue, published genomes in bold.

The cells of $C$. algicola are generally rod-shaped with rounded or tapered ends with cell lengths and widths ranging from 1.5 to 4 and 0.4 to 0.5 $\mu \mathrm{m}$, respectively (Figure 2 and Table 1). C. algicola is motile by gliding [1]. Colonies on marine 2216 agar have yellow-orange pigmentation and a compact center, with a spreading edge possessing lighter pigmentation. Their consistency is slimy and they are slightly sunken into the agar [1]. Flexirubin pigments are not formed. C. algicola grows between 0.5 and $10 \% \mathrm{NaCl}$, with the best growth in the presence of about $2 \% \mathrm{NaCl}$. The temperature range for growth is between $-2^{\circ} \mathrm{C}$ and $28^{\circ} \mathrm{C}$, with an optimum between $15-20^{\circ} \mathrm{C}$ on solid media and at about $20-25^{\circ} \mathrm{C}$ in liquid media [1]. The optimal $\mathrm{pH}$ for growth is about 7.5 [1].
The organism is strictly aerobic and chemoorganotrophic [1]. C. algicola can hydrolyze agar, starch, gelatine, carboxymethylcellulose (CMC), casein, Tween 80 , tributyrin and L-tyrosine, but not urate, xanthine or dextran, when grown in presence of 1\% L-tyrosine a reddish-brown diffusible pigment is formed [1]. Nitrate reduction is positive, whereas denitrification, $\mathrm{H}_{2} \mathrm{~S}$ production and indole production are negative $[1,18]$. Acid is formed oxidatively from D-galactose, D-glucose, Dfructose, sucrose, cellobiose, lactose and mannitol. Strain IC166 ${ }^{\mathrm{T}}$ is sensitive to ampicillin, streptomycin and carbenicillin and shows resistance to tetracycline [3]. 
Table 1. Classification and general features of C. algicola IC166 ${ }^{\top}$ according to the MIGS recommendations [13].

\begin{tabular}{llll}
\hline MIGS ID & \multicolumn{1}{c}{ Property } & \multicolumn{1}{c}{ Term } & Evidence code \\
\hline & & Domain Bacteria & TAS [14] \\
& & Phylum Bacteroidetes & TAS [15,16] \\
& Class Flavobacteria & TAS [1] \\
& & Order 'Flavobacteriales & TAS [15] \\
& & Family Flavobacteriaceae & TAS [18-21] \\
& & Genus Cellulophaga & TAS [2] \\
& Gram stain & Type strain IC166 & TAS [1] \\
& Cell shape & negative & TAS [1] \\
& Motility & rod-shaped & TAS [1] \\
& Sporulation & motile by gliding & TAS [1] \\
& Temperature range & none & TAS [1] \\
& Optimum temperature & 20 ${ }^{\circ}$ C $-28^{\circ}$ C & TAS [1] \\
& Salinity & 0.5-10\% NaCl & TAS [1] \\
MIGS-22 & Oxygen requirement & aerobic & TAS [1] \\
& Carbon source & carbohydrates & TAS [1] \\
& Energy source & chemoheterotroph & TAS [1] \\
MIGS-6 & Habitat & sea ice diatoms, macrophyte surfaces & TAS [1] \\
MIGS-15 & Biotic relationship & free-living & TAS [1] \\
MIGS-14 & Pathogenicity & none & NAS \\
& Biosafety level & 1 & NAS \\
& Isolation & surfaces of Antarctic algae & TAS [22] \\
MIGS-4 & Geographic location & eastern Antarctic coastal zone & TAS [1] \\
MIGS-5 & Sample collection time & 1996 & TAS [1] \\
MIGS-4.1 & Latitude & not reported & NAS \\
MIGS-4.2 & Longitude & not reported & NAS \\
MIGS-4.3 & Depth & not reported & NAS \\
MIGS-4.4 & Altitude & not reported & NAS \\
\hline & & NAS \\
\hline
\end{tabular}

Evidence codes - IDA: Inferred from Direct Assay (first time in publication); TAS: Traceable Author Statement (i.e., a direct report exists in the literature); NAS: Non-traceable Author Statement (i.e., not directly observed for the living, isolated sample, but based on a generally accepted property for the species, or anecdotal evidence). These evidence codes are from of the Gene Ontology project [23]. If the evidence code is IDA, then the property was directly observed by one of the authors or an expert mentioned in the acknowledgements.

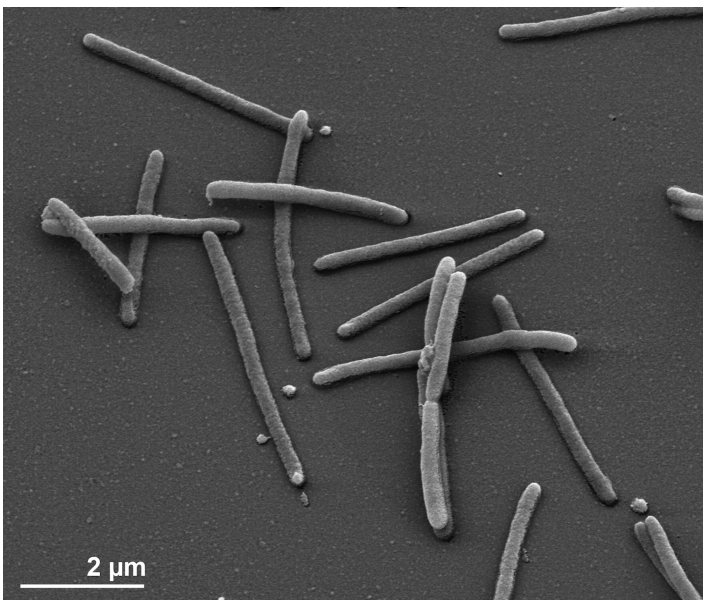

Figure 2. Scanning electron micrograph of C. algicola IC166 ${ }^{\top}$ 


\section{Chemotaxonomy}

The fatty acid profile of seven Antarctic strains, including strain IC166 ${ }^{\mathrm{T}}$, was analyzed by Bowman in 2000 [1]. The hypothetical median representative of the Antarctic isolates was published. The predominant cellular fatty acids of these seven strains were branched-chain saturated and unsaturated fatty acids and straight-chain saturated and mono-unsaturated fatty acids, namely iso- $\mathrm{C}_{15: 0}$ (7.5\%), iso- $\mathrm{C}_{15: 1 \omega 10 \mathrm{c}}(7.5 \%)$, iso $-\mathrm{C}_{17: 1 \omega 7 \mathrm{c}}(6.1 \%)$, $\mathrm{C}_{15: 0}(14.3 \%), \mathrm{C}_{16: 1 \omega 7 \mathrm{c}}(19.2 \%)$, iso $-\mathrm{C}_{15: 0}$ 3-он $(8.6 \%)$, iso- $\mathrm{C}_{16: 0}$ 3-он $(6.5 \%)$ and iso $-\mathrm{C}_{17: 0}$ 3-он $(4.5 \%)$ [1]. The isoprenoid quinones of $C$. algicola were not determined, but for $C$. pacifica the presence of MK6 as the major lipoquinone was described [3]. Polar lipids not have been studied.

\section{Genome sequencing and annotation Genome project history}

This organism was selected for sequencing on the basis of its phylogenetic position [24], and is part of the Genomic Encyclopedia of Bacteria and Archaea project [25]. The genome project is deposited in the Genomes OnLine Database [12] and the complete genome sequence is deposited in GenBank. Sequencing, finishing and annotation were performed by the DOE Joint Genome Institute (JGI). A summary of the project information is shown in Table 2.

Table 2. Genome sequencing project information

\begin{tabular}{lll}
\hline MIGS ID & Property & Term \\
\hline MIGS-31 & Finishing quality & Finished \\
MIGS-28 & Libraries used & Three genomic libraries: one 454 pyrosequence standard library, one \\
MIGS-29 & Sequencing platforms & Iflumina GAii, 454 GS FLX Titanium \\
MIGS-31.2 & Sequencing coverage & $146.0 \times$ Illumina; $53.5 \times$ pyrosequence \\
MIGS-30 & Assemblers & Newbler version 2.0.00.20-PostRelease-10-28-2008-g-3.4.6, Velvet \\
& Gersion 0.7.63, phrap version SPS D 4.24 \\
MIGS-32 & Gene calling method & Prodigal 1.4, GenePRIMP \\
& INSDC ID & CP002453 \\
& Genbank Date of Release & January 18, 2011 \\
& GOLD ID & Gc01592 \\
& NCBI project ID & 41529 \\
& Database: IMG-GEBA & 2503904003 \\
MIGS-13 & Source material identifier & DSM 14237 \\
& Project relevance & Tree of Life, GEBA \\
\hline
\end{tabular}

\section{Growth conditions and DNA isolation}

C. algicola IC166 ${ }^{\mathrm{T}}$, DSM 14237, was grown in DSMZ medium 514 (BACTO marine broth) [26] at $15^{\circ} \mathrm{C}$. DNA was isolated from $0.5-1 \mathrm{~g}$ of cell paste using MasterPure Gram-positive DNA purification kit (Epicentre MGP04100) following the standard protocol as recommended by the manufacturer with modification st/DL for cell lysis as described in Wu et al. [25]. DNA is available through the DNA Bank Network [27].

\section{Genome sequencing and assembly}

The genome was sequenced using a combination of Illumina and 454 sequencing platforms. All general aspects of library construction and sequencing can be found at the JGI website [28]. Py- rosequencing reads were assembled using the Newbler assembler version 2.3-PreRelease-09-142009-bin (Roche). The initial Newbler assembly consisting of 128 contigs in two scaffolds was converted into a phrap assembly by [29] making fake reads from the consensus, to collect the read pairs in the 454 paired end library. Illumina GAii sequencing data $(710 \mathrm{Mb})$ was assembled with Velvet [30] and the consensus sequences were shredded into $1.5 \mathrm{~kb}$ overlapped fake reads and assembled together with the 454 data. The 454 draft assembly was based on $263.4 \mathrm{Mb} 454$ draft data and all of the 454 paired end data. Newbler parameters are -consed -a 50 -l 350 -g -m -ml 20. The Phred/Phrap/Consed software package [29] 
was used for sequence assembly and quality assessment in the subsequent finishing process. After the shotgun stage, reads were assembled with parallel phrap (High Performance Software, LLC). Possible mis-assemblies were corrected with gapResolution [28], Dupfinisher [31], or sequencing cloned bridging PCR fragments with subcloning or transposon bombing (Epicentre Biotechnologies, Madison, WI). Gaps between contigs were closed by editing in Consed, by PCR and by Bubble PCR primer walks (J.-F.Chang, unpublished). A total of 1,054 additional reactions and three shatter libraries were necessary to close gaps and to raise the quality of the finished sequence. Illumina reads were also used to correct potential base errors and increase consensus quality using a software Polisher developed at JGI [32]. The error rate of the completed genome sequence is less than 1 in 100,000 . Together, the combination of the Illumina and 454 sequencing platforms provided 199.5 $x$ coverage of the genome. The final assembly contained 697,305 pyrosequence and 20,331,123 Illumina reads

\section{Genome annotation}

Genes were identified using Prodigal [33] as part of the Oak Ridge National Laboratory genome annotation pipeline, followed by a round of manual curation using the JGI GenePRIMP pipeline [34]. The predicted CDSs were translated and used to search the National Center for Biotechnology Information (NCBI) nonredundant database, UniProt, TIGR-Fam, Pfam, PRIAM, KEGG, COG, and InterPro databases. Additional gene prediction analysis and functional annotation was performed within the Integrated Microbial Genomes - Expert Review (IMG-ER) platform [35].

\section{Genome properties}

The genome consists of a 4,888,353 bp long chromosome with a GC content of $33.8 \%$ (Table 3 and Figure 3). Of the 4,347 genes predicted, 4,285 were protein-coding genes, and 62 RNAs; 122 pseudogenes were also identified. The majority of the protein-coding genes (59.5\%) were assigned with a putative function while the remaining ones were annotated as hypothetical proteins. The distribution of genes into COGs functional categories is presented in Table 4.

Table 3. Genome Statistics

\begin{tabular}{lrr}
\hline Attribute & Value & \% of Total \\
\hline Genome size (bp) & $4,888,353$ & $100.00 \%$ \\
DNA coding region (bp) & $4,301,528$ & $88.00 \%$ \\
DNA G+C content (bp) & $1,650,610$ & $33.77 \%$ \\
Number of replicons & 1 & \\
Extrachromosomal elements & 0 & \\
Total genes & 4,347 & $100.00 \%$ \\
RNA genes & 62 & $1.43 \%$ \\
rRNA operons & 5 & \\
Protein-coding genes & 4,285 & $98.57 \%$ \\
Pseudo genes & 122 & $2.81 \%$ \\
Genes with function prediction & 2,587 & $59.51 \%$ \\
Genes in paralog clusters & 698 & $16.06 \%$ \\
Genes assigned to COGs & 2,539 & $58.41 \%$ \\
Genes assigned Pfam domains & 2,822 & $64.92 \%$ \\
Genes with signal peptides & 1,220 & $28.07 \%$ \\
Genes with transmembrane helices & 1,010 & $23.23 \%$ \\
CRISPR repeats & 0 & \\
\hline
\end{tabular}




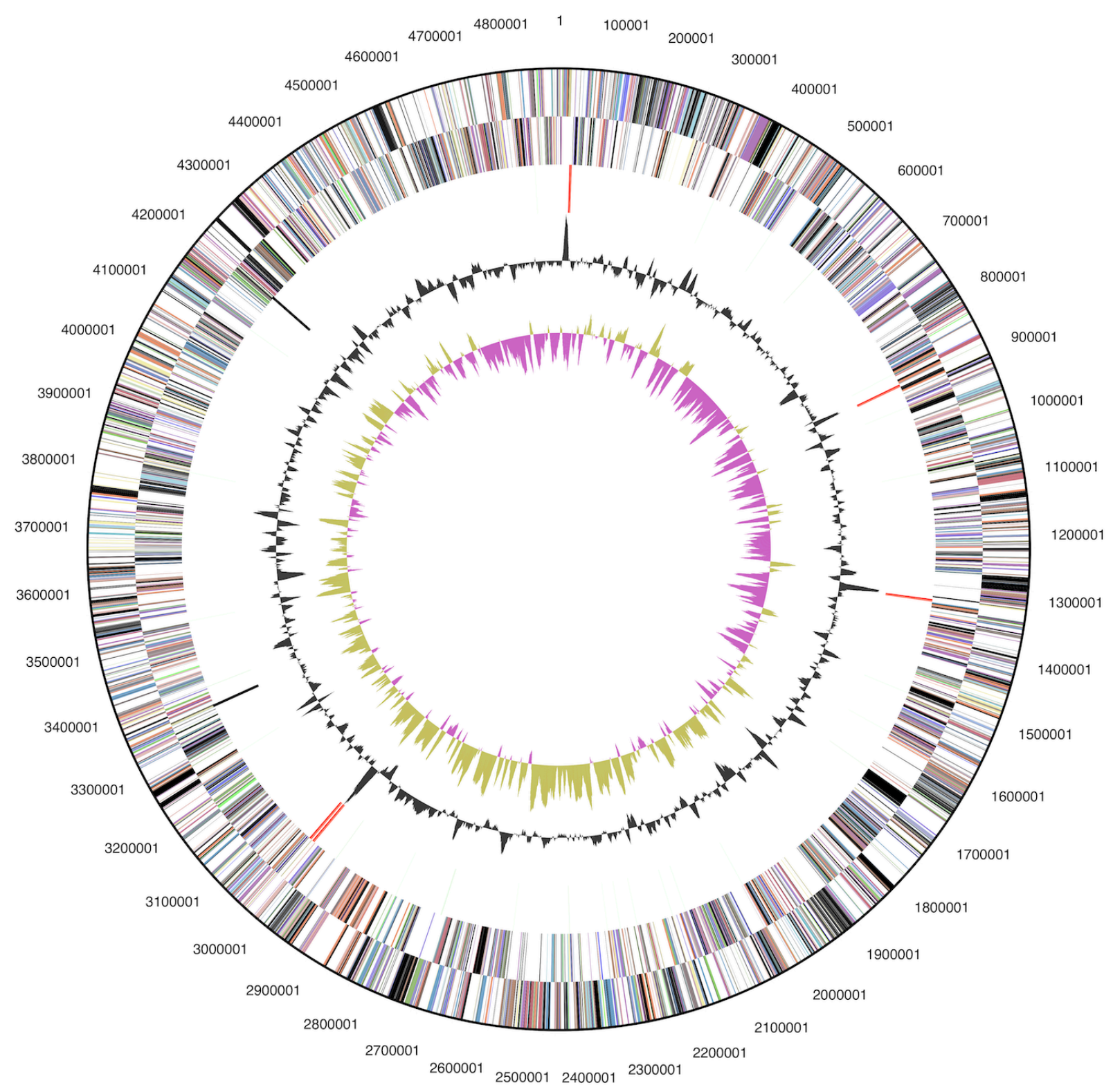

Figure 3. Graphical circular map of the chromosome. From outside to the center: Genes on forward strand (color by COG categories), Genes on reverse strand (color by COG categories), RNA genes (tRNAs green, rRNAs red, other RNAs black), GC content, GC skew.

\section{Insights from genome sequence}

A closer look on the genome sequence of strain IC166 ${ }^{\mathrm{T}}$ revealed a set of genes which might be responsible for the yellow-orange color of $C$. algicola cells by encoding enzymes that are involved in the synthesis of carotenoids. Carotenoids are produced by the action of geranylgeranyl pyrophosphate synthase (Celal_1770), phytoene synthase (Celal_2446), phytoene desaturase (Celal_2447), lycopene cyclase (Celal_1771) and carotene hydroxylase (Celal_2445). Geranylgeranyl pyrophosphate synthases start the biosynthesis of ca- rotenoids by combining farnesyl pyrophosphate with $\mathrm{C}_{5}$ isoprenoid units to $\mathrm{C}_{20}$-molecules, geranylgeranyl pyrophosphate. The phytoene synthase catalyzes the condensation of two geranylgeranyl pyrophosphate molecules followed by the removal of diphosphate and a proton shift leading to the formation of phytoene. Sequential desaturation steps are conducted by the phytoene desaturase followed by cyclisation of the ends of the molecules catalyzed by the lycopene cyclase [36]. 


\begin{tabular}{lrrl}
\multicolumn{5}{l}{ Table 4. Number of genes associated with the general COG functional categories } \\
\hline Code & value & \%age & Description \\
\hline J & 160 & 5.8 & Translation, ribosomal structure and biogenesis \\
A & 0 & 0.0 & RNA processing and modification \\
K & 174 & 6.3 & Transcription \\
L & 147 & 5.4 & Replication, recombination and repair \\
B & 1 & 0.0 & Chromatin structure and dynamics \\
D & 20 & 0.7 & Cell cycle control, cell division, chromosome partitioning \\
Y & 0 & 0.0 & Nuclear structure \\
V & 63 & 2.3 & Defense mechanisms \\
T & 167 & 6.1 & Signal transduction mechanisms \\
M & 239 & 8.7 & Cell wall/membrane/envelope biogenesis \\
N & 7 & 0.3 & Cell motility \\
Z & 0 & 0.0 & Cytoskeleton \\
W & 0 & 0.0 & Extracellular structures \\
U & 41 & 1.5 & Intracellular trafficking, secretion, and vesicular transport \\
O & 99 & 3.6 & Posttranslational modification, protein turnover, chaperones \\
C & 135 & 4.9 & Energy production and conversion \\
G & 172 & 6.3 & Carbohydrate transport and metabolism \\
E & 208 & 7.6 & Amino acid transport and metabolism \\
F & 70 & 2.6 & Nucleotide transport and metabolism \\
H & 131 & 4.8 & Coenzyme transport and metabolism \\
I & 97 & 3.5 & Lipid transport and metabolism \\
P & 174 & 6.3 & Inorganic ion transport and metabolism \\
Q & 52 & 1.9 & Secondary metabolites biosynthesis, transport and catabolism \\
R & 345 & 12.6 & General function prediction only \\
S & 247 & 9.0 & Function unknown \\
- & 1,808 & 41.6 & Not in COGs \\
\hline & & &
\end{tabular}

Strain IC166 ${ }^{\mathrm{T}}$ produces a wide range of extracellular enzymes degrading proteins and polysaccharides. These enzymes are cold adapted, they have temperature optima between $15-30^{\circ} \mathrm{C}$ and can tolerate temperatures below $0^{\circ} \mathrm{C}$ [37]. For that reason they are of special interest for industrial and biotechnical applications. C. algicola like the other members of the genus Cellulophaga, cannot hydrolyze filter paper or cellulose in its crystalline form, though they can hydrolyze the soluble cellulose derivative carboxymethylcellulose (CMC). The genome sequence of strain IC166 ${ }^{\mathrm{T}}$ revealed the presence of three cellulases (Celal_0025, Celal_2753, Celal_3912), probably responsible for the hydrolysis of CMC. In addition two $\beta$-glucosidases (Celal_0470, Celal_1802) were identified in the genome, catalyzing the break down of the glycosidic $\beta-1,4$ bond between two glucose molecules in cellobiose.
The IC166 ${ }^{\mathrm{T}}$ genome contains 22 genes coding for sulfatases, which are located in close proximity to glycoside hydrolase genes suggesting that sulfated polysaccharides may be used as substrates. $\alpha$-Lfucoidan could be a substrate, as five $\alpha$-Lfucosidases (Celal_2459, Celal_2466, Celal_2469, Celal_2470, Celal_2473) are located in close proximity to three sulfatases (Celal_2464, Celal_2468, Celal_2472). Sakai and colleagues report the existence of intracellular $\alpha$-L-fucosidases and sulfatases, which enable 'Fucophilus fucoidanolyticus' to degrade fucoidan [38]. This fucoidan degrading ability could be also shared by Coraliomargarita akajimensis, as the annotation of the genome sequence revealed the existence of 49 sulfatases and twelve $\alpha$-L-fucosidases [39]. In addition, three $\beta$ agarases (Celal_2463, Celal_2494, Celal_3979) were identified, with two of them located in the above mentioned region, which is rich in genes encoding glycoside hydrolases and sulfatases. 


\section{Acknowledgements}

We would like to gratefully acknowledge the help of Regine Fähnrich (DSMZ) for growing C. algicola cultures. This work was performed under the auspices of the US Department of Energy Office of Science, Biological and Environmental Research Program, and by the University of California, Lawrence Berkeley National Laboratory under contract No. DE-

\section{References}

1. Bowman JP. Description of Cellulophaga algicola sp. nov., isolated from the surfaces of Antarctic algae, and reclassification of Cytophaga uliginosa (ZoBell and Upham 1944) Reichenbach 1989 as Cellulophaga uliginosa comb. nov. Int I Syst Evol Microbiol 2000; 50:1861-1868. PubMed

2. Johansen JE, Nielsen P, Sjøholm C. Description of Cellulophaga baltica gen. nov., sp. nov. and Cellulophaga fucicola gen. nov., sp. nov. and reclassification of [Cytophaga] lytica to Cellulophaga lytica gen. nov., comb. nov. Int I Syst Evol Microbiol 1999; 49:1231-1240. PubMed

3. Nedashkovskaya OI, Suzuki M, Lysenko AM, Snauwaert C, Vancanneyt M, Swings J, Vysotskii MV, Mikhailov VV. Cellulophaga pacifica sp. nov. Int J Syst Evol Microbiol 2004; 54:609-613. PubMed doi:10.1099/ijs.0.02737-0

4. Kahng HY, Chung BS, Lee DH, Jung JS, Park JH, Joen CO. Cellulophaga tyrosinoxydans sp. nov., a tyrosinase producing bacterium isolated from seawater. Int J Syst Evol Microbiol 2009; 59:654-657. PubMed doi:10.1099/ijs.0.003210-0

5. DeSantis TZ, Hugenholtz P, Larsen N, Rojas M, Brodie EL, Keller K, Huber T, Dalevi D, Hu P, Andersen GL. Greengenes, a chimera-checked $16 \mathrm{~S}$ rRNA gene database and workbench compatible with ARB. Appl Environ Microbiol 2006; 72:50695072. PubMed doi:10.1128/AEM.03006-05

6. Porter MF. An algorithm for suffix stripping. Program: electronic library and information systems 1980; 14:130-137 doi:10.1108/eb046814

7. Venter JC, Remington K, Heidelberg JF, Halpern AL, Rusch D, Eisen JA, Wu D, Paulsen I, Nelson $\mathrm{KE}$, Nelson W. Environmental genome shotgun sequencing of the Sargasso Sea. Science 2004; 304:66-74. PubMed doi:10.1126/science.1093857

8. Castresana J. Selection of conserved blocks from multiple alignments for their use in phylogenetic analysis. Mol Biol Evol 2000; 17:540-552. PubMed

9. Lee C, Grasso C, Sharlow MF. Multiple sequence alignment using partial order graphs. Bioinformatics 2002; 18:452-464. PubMed doi:10.1093/bioinformatics/18.3.452
AC02-05CH11231, Lawrence Livermore National Laboratory under Contract No. DE-AC52-07NA27344, and Los Alamos National Laboratory under contract No. DE-AC0206NA25396, UT-Battelle and Oak Ridge National Laboratory under contract DE-AC05-000R22725, as well as German Research Foundation (DFG) INST 599/1-2.

10. Stamatakis $A$, Hoover $P$, Rougemont J. A rapid bootstrap algorithm for the RAxML Web servers. Syst Biol 2008; 57:758-771. PubMed doi:10.1080/10635150802429642

11. Pattengale ND, Alipour M, Bininda-Emonds ORP, Moret BME, Stamatakis A. How many bootstrap replicates are necessary? Lect Notes Comput Sci 2009; 5541:184-200. doi:10.1007/978-3-642$\underline{02008-7 \quad 13}$

12. Liolios K, Chen IM, Mavromatis K, Tavernarakis N, Hugenholtz P, Markowitz VM, Kyrpides NC. The Genomes On Line Database (GOLD) in 2009: status of genomic and metagenomic projects and their associated metadata. Nucleic Acids Res 2010; 38:D346-D354. PubMed doi:10.1093/nar/gkp848

13. Field D, Garrity G, Gray T, Morrison N, Selengut J, Sterk P, Tatusova T, Thomson N, Allen MJ, Angiuoli SV, et al. The minimum information about a genome sequence (MIGS) specification. Nat Biotechnol 2008; 26:541-547. PubMed doi:10.1038/nbt1360

14. Woese CR, Kandler O, Wheelis ML. Towards a natural system of organisms: proposal for the domains Archaea, Bacteria, and Eucarya. Proc Natl Acad Sci USA 1990; 87:4576-4579. PubMed doi:10.1073/pnas.87.12.4576

15. Garrity GM, Holt J. Taxonomic outline of the Archaea and Bacteria. In: Bergey's Manual of Systematic Bacteriology, $2^{\text {nd }}$ ed. vol. 1. The Archaea, Deeply Branchingand Phototrophic Bacteria. Garrity GM, Boone DR and Castenholz RW (eds). 2001; 155-166.

16. Garrity GM, Holt JG. The Road Map to the Manual. In: Garrity GM, Boone DR, Castenholz RW (eds), Bergey's Manual of Systematic Bacteriology, Second Edition, Volume 1, Springer, New York, 2001, p. 119-169.

17. Ludwig W, Euzeby J, Whitman WG. Draft taxonomic outline of the Bacteroidetes, Planctomycetes, Chlamydiae, Spirochaetes, Fibrobacteres, Fusobacteria, Acidobacteria, Verrucomicrobia, Dictyoglomi, and Gemmatimonadetes. http://www.bergeys.org/outlines/Bergeys_Vol_4_O utline.pdf. Taxonomic Outline 2008. 
18. Bernardet JF, Nakagawa Y, Holmes B. Proposed minimal standards for describing new taxa of the family Flavobacteriaceae and emended description of the family. Int I Syst Evol Microbiol 2002; 52:1049-1070. PubMed doi:10.1099/ijs.0.02136-0

19. List Editor. Validation of the publication of new names and new combinations previously effectively published outside the IJSB. List No. 41. Int J Syst Bacteriol 1992; 42:327-328. doi:10.1099/00207713-42-2-327

20. Reichenbach H. Order 1. Cytophagales Leadbetter 1974, 99AL. In: Holt JG (ed), Bergey's Manual of Systematic Bacteriology, First Edition, Volume 3, The Williams and Wilkins Co., Baltimore, 1989, p. 2011-2013.

21. Bernardet JF, Segers $P$, Vancanneyt $M$, Berthe $F$, Kersters K, Vandamme P. Cutting a Gordian knot: emended classification and description of the genus Flavobacterium, emended description of the family Flavobacteriaceae, and proposal of Flavobacterium hydatis nom. nov. (Basonym, Cytophaga aquatilis Strohl and Tait 1978). Int J Syst Bacteriol 1996; 46:128-148. doi:10.1099/00207713-46-1-128

22. Classification of. Bacteria and Archaea in risk groups. http://www.baua.de.

23. Ashburner M, Ball CA, Blake JA, Botstein D, Butler H, Cherry JM, Davis AP, Dolinski K, Dwight SS, Eppig JT, et al. Gene Ontology: tool for the unification of biology. Nat Genet 2000; 25:25-29. PubMed doi:10.1038/75556

24. Klenk HP, Goeker M. En route to a genome-based classification of Archaea and Bacteria? Syst Appl Microbiol 2010; 33:175-182. PubMed doi:10.1016/j.syapm.2010.03.003

25. Wu D, Hugenholtz P, Mavromatis K, Pukall R, Dalin E, Ivanova NN, Kunin V, Goodwin L, Wu M, Tindall BJ, et al. A phylogeny-driven genomic encyclopaedia of Bacteria and Archaea. Nature 2009; 462:1056-1060. PubMed doi:10.1038/nature08656

26. List of growth media used at DSMZ: http://www.dsmz.de/microorganisms/media list.ph p.

27. Gemeinholzer B, Dröge $G$, Zetzsche $H$, Haszprunar G, Klenk HP, Güntsch A, Berendsohn WG, Wägele JW. The DNA Bank Network: the start from a German initiative. Biopreservation and Biobanking (In press).

28. The DOE Joint Genome Institute. www.jgi.doe.gov

29. Phrap and Phred for Windows. MacOS, Linux, and Unix. http://www.phrap.com
30. Zerbino DR, Birney E. Velvet: algorithms for de novo short read assembly using de Bruijn graphs. Genome Res 2008; 18:821-829. PubMed doi:10.1101/gr.074492.107

31. Han C, Chain P. 2006. Finishing repeat regions automatically with Dupfinisher. in Proceeding of the 2006 international conference on bioinformatics \& computational biology. Edited by Hamid R. Arabnia \& Homayoun Valafar, CSREA Press. June 26-29, 2006: 141-146.

32. Lapidus A, LaButti K, Foster B, Lowry S, Trong S, Goltsman E. POLISHER: An effective tool for using ultra short reads in microbial genome assembly and finishing. AGBT, Marco Island, FL, 2008.

33. Hyatt $\mathrm{D}$, Chen $\mathrm{GL}$, LoCascio PF, Land $\mathrm{ML}$, Larimer FW, Hauser LJ. Prodigal: prokaryotic gene recognition and translation initiation site identification. BMC Bioinformatics 2010; 11:119. PubMed doi:10.1186/1471-2105-11-119

34. Pati A, Ivanova NN, Mikhailova N, Ovchinnikova G, Hooper SD, Lykidis A, Kyrpides NC. GenePRIMP: a gene prediction improvement pipeline for prokaryotic genomes. Nat Methods 2010; 7:455-457. PubMed doi:10.1038/nmeth.1457

35. Markowitz VM, Ivanova NN, Chen IMA, Chu K, Kyrpides NC. IMG ER: a system for microbial genome annotation expert review and curation. Bioinformatics 2009; 25:2271-2278. PubMed doi:10.1093/bioinformatics/btp393

36. Sandmann G. Carotenoid biosynthesis and biotechnological application. Arch Biochem Biophys 2001; 385:4-12. PubMed doi:10.1006/abbi.2000.2170

37. Nichols D, Bowman J, Sanderson K, Nichols CM, Lewis T, McMeekin T, Nichols PD. Developments with Antarctic microorganisms: culture collections, bioactivity screening, taxonomy, PUFA production and cold-adapted enzymes. Curr Opin Biotechnol 1999; 10:240-246. PubMed doi:10.1016/S0958$\underline{1669(99) 80042-1}$

38. Sakai T, Ishizuka K, Kato I. Isolation and characterization of fucoidan-degrading marine bacterium. Mar Biotechnol 2003; 5:409-416. PubMed doi:10.1007/s10126-002-0118-6

39. Mavromatis K, Abt B, Brambilla E, Lapidus A, Copeland A, Desphande S, Nolan M, Lucas S, Tice H, Cheng JF. Complete genome sequence of Coraliomargarita akajimensis type strain (04OKA010-24 T). Stand Genomic Sci 2010; 2:290-299. PubMed doi:10.4056/sigs.952166 